\title{
A CASE OF CHOREA INSANIENS WITH REPORT OF AUTOPSY.
}

BY ISABEL A. BRADLEY, M. D.,

Pathologist and Assistant Physician, Columbus State Hospital.

The case which I will present to you in this paper is one of unusual interest, not only because of the rarity of this mental state, but because it presents several pathological conditions which have been subjects of investigation for some time. Keenly realizing that the work is incomplete, I still believe that it is not entirely without value.

Mrs. E. R., age Ig, a native of Ohio, was admitted to the Columbus State Hospital, Nov. I I, 1902, and died at the Hospital Nov. 13, 1902, forty-three hours after admission. The medical certificate of her commitment papers gives the following evidences of insanity, "outbreaks of excitement, during which she is uncontrollable, delusions of persecution, unduly suspicious and at times incoherent."

The patient had not been well for about two weeks before coming to the hospital but had been cared for at her home. The only history obtainable concerning her parentage was that her mother was a very nervous and peculiar woman and was separated from her husband. There was no history of insanity or epilepsy in the family. The patient had always been nervous. This nervousness had increased since puberty. She was of a kindly disposition and always industrious. No history of previous sickness could be obtained. During the summer previous to her death she had nursed her mother through a long siege of typhoid fever. Seven weeks before her admission to the hospital she was married. She frequently complained of being tired, and gradually became more nervous and restless and slept very poorly. Several

' Read before the meeting of the Association of the Assistant Physicians of the Ohio State Hospitals, held in Toledo, Ohio, October 7-8, 1903. 
days before coming to the hospital she developed irregular, jerky movements of the body which were greatly exaggerated at times. At this time she had some control of herself, although occasionally this would be entirely lost. She also had convulsive seizures which were considered by her attending physician to be of the nature of hysteria major. She grew very suspicious of people and took a great dislike to doctors.

She was brought to the hospital about 4 P. M. Nov. II, I902. Her hair was down her back, her shoes off and she was only partially dressed. The movements of her body were such that she could neither sit nor stand. She would thrust first one hand and then the other through her hair. The trunk would bend to one side, then to the other and at times the whole body would give a twist. She would toss her head and jerk one limb and then the other.

She was conscious and orientation was normal. She knew that her friends thonght her insane.

Shortly after admission she took some bread and milk, and that evening went to the dining-room and ate some supper. She objected to going to bed and having a tray brought to her. Physical examination was impossible on account of the movements of the body. She appeared anæmic and had a rapid pulse. During the night she remained in bed fairly well but was restless and tossed about considerably, sleeping only a few moments at a time. The following day she was more restless and wanted to go home. She was quite incoherent and irritable. She took nourishment only when urged to do so. She was taken out-ofdoors during the day, as she appeared much quieter when out and was very anxious to go. That night when put to bed she began to toss about and very rapidly became worse. She would throw herself about, would sit up, then get on her knees or toss her head. She would throw her arms and legs about in such a way as to bruise them. She paid no attention to questioning and had absolutely no control of herself.

At 7.45 P. M. that evening she was put in a wet-sheet pack with cold cloths to her head. This did not quiet her movements. She continued to throw her head about and bite her tongue. At 9 o'clock she was taken out of the pack and restrained in bed. One-fiftieth of a grain of hyoscine hydrobromate was given 
hypodermically. The movements became more violent. She would scream, roll her eyes, protrude her tongue and quickly jerk it back, close her jaws firmly, blow through her nose forcibly, throw her limbs about and raise the trunk. These paroxysms would last from ten to fifteen seconds and would then be followed by hard breathing. Unless restrained the patient would injure her limbs by striking the edge of the bed. She was placed in a wet sheet wrung out of warm water and cold cloths were kept on the back of the neck and forehead. The face became flushed and for a time the changing of the cloths seemed to start the paroxysms. A small feeding tube was passed into the stomach and a pint of milk and a pint of water were introduced. Fifteen grains of chloral hydrate and thirty grains of bromide were also given.

At I.00 A. M. the motor symptoms still persisted, but the cryingout had ceased. About 3 A. M. the motor symptoms subsided and the patient became comatose; the breathing was stertorous in character, the pulse was running and the temperature rising. At $6.30 \mathrm{~A}$. M. the temperature was $103 . \mathrm{I}^{\circ}$ per axilla, the pulse running and respiration irregular. At $8 \mathrm{~A}$. $\mathrm{M}$. the respirations were markedly Cheyne-Stokes. The patient died at II A. M.

I am indebted to Dr. G. T. Harding, Jr., the attending physician, for the clinical notes.

The autopsy was commenced two and one-half hours after death. The corpse was that of a young woman of small stature, well formed and with regular features. The nutrition was poor. The panniculus adiposus was very small in amount, the musculature small. Rigor mortis was appearing in the jaws. There was some hypostasis in the dependent parts. The eyes were prominent; the pupils widely dilated and equal. There were several abrasions on the lips where they had been bitten. The skin over the abdomen showed numerous superficial linear abrasions caused by the movements of her hands when in the pack. There were numerous black and blue spots on the leg. The fists were clenched and the feet extended.

Head.-The scalp was normal. The skull was thin. The dura was of normal thickness and not adherent to the calvarium. The longitudinal sinus was empty. The tension of the dura was markedly increased. The under surface was smooth and glisten- 
ing and was not adherent to the pia. The vessels of the pia were brightly injected. This was most marked in the posterior portion of the superior parietal regions. There was a considerable amount of clear, yellow fluid under the pia. The latter stripped from the convolutions easily. The consistence of the brain substance was considerably diminished. This was not so marked in the occipital regions. On section the gray matter had a glistening, swollen appearance, the natural divisions not being clearly defined. The blood-vessels throughout the brain substance were seen as bright red pin-points. The blood-vessels at the base of the brain were normal. The cerebro-spinal fluid was increased.

Thorax.-After the sternum was removed, in the upper part of the anterior mediastinum was a small pinkish body which in location and general contour corresponded to the thymus gland. It presented two well-defined lobes, the right lobe being about $2 \mathrm{~cm}$. longer that the left; it weighed a little over 8.5 grams and measured $5 \mathrm{~cm}$. in length, $4.5 \mathrm{~cm}$. in width and $.6 \mathrm{~cm}$. in thickness.

The heart weighed 185 grams and appeared small for the size of the body. In the pericardial sac there was about $15 \mathrm{cc}$. of clear, yellow fluid. The left ventricle was firmly contracted. The heart was empty, except that in the auricles there was a small amount of fluid blood. The auricular surface of the mitral flaps was thickly studded with small roundish, white vegetations which were firmly adherent to the underlying tissue. The aorta, pulmonary and tri-cuspid valves were normal. The heart-muscle was normal in appearance.

The left lung weighed 160 grams and was alherent to the chest wall and diaphragm; the adhesions were easily torn. Otherwise the lung was normal.

The right lung weighed 250 grams and, aside from a small deeply-congested area in the posterior middle portion of the upper lobe, was normal in appearance. There were several small, caseous bronchial glands.

Abdomen.-The peritoneum had a dry, dull appearance. The position of the organs was normal. The spleen weighed I25 grams. The capsule was smooth and distended. On section the pulp was increased somewhat. The stroma was fairly distinct and the follicles slightly increased in prominence. 
The kidneys weighed 120 grams each and but for deeply congested pyramids, appeared normal. The liver weighed IIgO grams. There was an increased amount of blood in the parenchyma.

The pancreas and adrenals appeared normal. The stomach contained about $250 \mathrm{cc}$. of a dark greenish fluid with a few semisolid particles having the odor of sour bile. The mucosa of both stomach and intestines was pale but otherwise normal.

The uterus was pregnant and contained a fœtus $4.5 \mathrm{~cm}$. long and was probably about ten weeks old. The ovaries were of normal size. The right ovary contained a corpus luteum verum. The cortices of both ovaries contained Graaffian follicles in different stages of development. The bladder contained about $60 \mathrm{cc}$. of dark turbid urine. The mucosa was pale.

The mesenteric lymph-glands were markedly enlarged. The inguinal and axillary glands were of normal size. The hemolymph glands presented a pinkish color, and one, a bright red color.

The aorta was small, measuring only $3.25 \mathrm{~cm}$. in circumference in the abdominal poition. The intima was smooth and glistening, with no areas of degeneration. The elasticity was normal.

Anatomical diagnosis.-Edema of the brain, pregnant uterus, persistent thymus and partial lymphatic hyperplasia, arterial hypoplasia, organized mitral vegetations, congestion of the liver, spleen and kidneys; caseous bronchial glands.

Microscopic examination.-The kidneys and liver showed an active congestion but were otherwise normal. In the spleen there was an endothelial hyperplasia throughout, which was most marked in the centers of the Malpighian follicles. The center of some of the follicles was becoming hyaline.

The mesenteric lymph-glands showed an endothelial hyperplasia. The hemorrhagic area in the lung had a thin connective-tissue wall which separated it from the surrounding lung tissue. The heart-muscle, pancreas and adrenals were normal.

The appearance of the thymus was that of an actively functioning organ. The adenoid tissue was abundant and made up of dense masses of lymphoid cells with very deeply-staining nuclei. The characteristic bodies of thymic tissue were numerous. Only one Hassel's corpuscle was seen which showed calcareous de- 
generation. There was some congestion of the blood-vessels in the interlobular connective tissue. The hemolymph gland examined had its sinus and blood-vessels distended with red bloodcells.

Cortex.-With the naked eye the sections of the cortex could be seen to contain many small, clear spaces, both in the white and gray matter of the cerebrum. In the cerebellum they were confined to the white matter. In the sections examined these appeared to be somewhat more numerous in the central and parietal regions than in the frontal convolutions. These clear spaces were for the most part round and the largest were fully $5 \mathrm{~mm}$. in diameter. Under the microscope the largest spaces appeared to be independent of the blood-vessels, but the smaller openings were due to dilated perivascular and pericellular lymph-spaces.

$A$ section from the lower portion of the left posterior central convolution.-Large pyramidal cells. The protoplasm was very faintly and diffusely stained but the outline of the cells was distinctly made out. Many cells contained vacuoles in the periphery of the protoplasm. Some cells were mere shadows. The processes were very distinctly visible for a long distance and were stained a diffuse blue.

The nuclear membrane was entirely absent in many cells, the nuclei being indistinguishable from the surrounding cell-body. The nucleoli were very deeply stained, of about the normal size, usually normally located and many contained light areas.

The outline of the smaller pyramidal cells of the second layer was in most cases not distinguishable. The cells appeared to be disappearing and all the evidences of degeneration were in a more advanced stage than in the largest pyramidals. The cells of the frontal convolutions were similar to those in the central convolutions except that the protoplasm was more faintly stained and absolutely homogeneous; the nucleoli being small and very densely stained and having the appearance of being nearer the eye than the surrounding protoplasm. This was due to the advanced stage of degeneration of the protoplasm. The changes in the occipital lobe were not so advanced; the outline of the cells was distinct; the nuclei were definable, although the membrane was not visible and the nucleoli did not contain light areas. There were no nuclear folds in any of the sections examined. 
The neuroglia did not appear to be increased in amount, although no special neurogliar stains were used. Several neuroglia nuclei were very deeply stained and had a notched outline similar to those described by Hoch.

Pieces were taken from the first and second frontal convolutions of both sides of the brain, left central convolution, right superior parietal, right occipital, lenticular nucleus and cerebellum and stained by Nissl's methylene-blue method and hematoxylin and eosin.

The spinal cord was not examined.

Summary of the examination of the cortex.-The changes in the cells were fairly constant, although more marked in the central, parietal, and frontal convolutions. The second and third layers of the cortex seemed to have suffered the most. The changes consisted in a diffuse chromatolysis of the entire cell, the protoplasm taking a very light blue and having a crumbly appearance. The processes were abnormally visible, due to the achromatic substance taking the stain. The nuclei were small and the nuclear membrane was entirely absent. The nucleoli contained light areas and were usually small. The smaller pyramidal cells of the second layer showed the most advanced changes. The perivascular and pericellular dilatations were most extensive in the parietal and central convolutions. The very large, clear spaces were not seen in the sections of the lenticular nucleus or the cerebellum, but in these localities there were numerous perivascular dilatations.

It is greatly regretted that no bacteriological examination was made in this case, nevertheless, since there was no evidence of a gas-producing organism being present in any other organ of the body it does not seem likely that the openings in the brain substance were due to such a cause. It is most probable that there was a genuine œdema of the brain. Although there was a hyperæmic condition of the meninges and brain substance, there was no inflammatory condition. The elevation of temperature might have been due to the œdematous condition of the corpus striatum, for there is considerable evidence that lesions in the corpus striatum and optic thalamus produce elevation of

2American Journal of Insanity, Vol. 54. 
bodily temperature." Mitchinson," reports two cases of rapidly fatal maniacal chorea and in both cases there was œdema of the brain. Bertram Abrahams "describes the cortical changes in a fatal case of acute chorea. They were very similar to those found in this present instance. He says, "The perivascular and perineuronal lymph-spaces were much dilated and the cortex was distinctly œedematous." Dana, describes a case of chorea in which the white matter of the brain was honey-combed with little spaces.

The points to be emphasized in the clinical history are a neurotic hereditary taint, a nervous temperament, physical exhaustion followed by the connubial state, delusions of persecutions, hysterical phenomena, choreic movements, incoherence, delirium and coma.

Summary of pathological findings. - Edema of the brain with advanced degeneration of its most highly specialized elements, the ganglion cells; organized mitral vegetations, congested hemolymph glands, arterial hypoplasia, an apparently actively functioning persistent thymus and mesenteric lymphatic hyperplasia.

The mental symptoms developed before the chorea but were very soon followed by the hysterical phenomena and the choreic movements. That the attacks in which the patient screamed, ran her tongue in and out, forcibly blew her nose, rolled her eyes about and raised the trunk of her body from the bed, were different from the general and constant twitching and throwing of her limbs and body can not be denied. The former no doubt belonged to a hysteria which accompanied the chorea. The gravid uterus was a predisposing factor in the production of the chorea. Often those who become choreic as a complication of pregnancy have suffered from chorea or rheumatic fever in youth. The absence of rheumatism or other infectious diseases from the history and the presence of the organized vegetations on the flaps of the mitral valve is significant.

The presence in this case of the lymphatic constitution as described by Ohlmacher,' is interesting and suggestive. While

- Lancet, June 29, 1889, p. 1395.

- Lancet, May II, I889.

- Lancet, February 10, 1900.

- Brain, I890.

The Bulletin of the Ohio Hospital for Epileptics, Vol. I, Nos. 2 and 3. 
the picture is not as pronounced as in most of his cases, yet the apparently actively functioning, persistent thymus and the hyperplasia of the mesenteric lymphatics with a small aorta is, I think, sufficient to consider this a case of constitutio lymphatica.

The lymphatic constitution may be an anatomical evidence of a neurotic constitution, but the cause of the hyperplasia of one set of organs and the hypoplasia of another set of organs is still unknown. That a toxic agent is concerned seems most probable and that the condition is an auto-intoxication due to a disordered metabolism is also very probable. I believe the same poison in the system which caused the endocardial lesions, the changes in the spleen and the congested internal organs, in the exhausted and highly susceptible organism, caused the cedema of the brain and the changes in the cortical cells and produced the terrific mental and motor phenomena which we saw fit to call maniacal chorea.

Our pathological studies will permit us to see the ravages of this poison which is circulating in the fluids of the body, but we must carry our investigations into the domain of chemistry before the real cause can be determined. 